\title{
Active Fault-Tolerant Control for Wind Turbine with Simultaneous Actuator and Sensor Faults
}

\author{
Lei Wang, ${ }^{1,2}$ Ming Cai, ${ }^{1,2}$ Hu Zhang, ${ }^{1,2}$ Fuad Alsaadi, ${ }^{3}$ and Liu Chen ${ }^{4}$ \\ ${ }^{1}$ Key Laboratory of Dependable Service in Cyber Physical Society, Ministry of Education, Chongqing University, \\ Chongqing 400044, China \\ ${ }^{2}$ College of Automation, Chongqing University, Chongqing 400044, China \\ ${ }^{3}$ Department of Electrical and Computer Engineering, Faculty of Engineering, King Abdulaziz University, \\ Jeddah 21589, Saudi Arabia \\ ${ }^{4}$ Chongqing Keneng Senior Technical Institute, Chongqing 400044, China
}

Correspondence should be addressed to Lei Wang; leiwang08@cqu.edu.cn

Received 4 May 2017; Revised 2 August 2017; Accepted 7 November 2017; Published 28 December 2017

Academic Editor: Roberto Natella

Copyright (C) 2017 Lei Wang et al. This is an open access article distributed under the Creative Commons Attribution License, which permits unrestricted use, distribution, and reproduction in any medium, provided the original work is properly cited.

\begin{abstract}
The purpose of this paper is to show a novel fault-tolerant tracking control (FTC) strategy with robust fault estimation and compensating for simultaneous actuator sensor faults. Based on the framework of fault-tolerant control, developing an FTC design method for wind turbines is a challenge and, thus, they can tolerate simultaneous pitch actuator and pitch sensor faults having bounded first time derivatives. The paper's key contribution is proposing a descriptor sliding mode method, in which for establishing a novel augmented descriptor system, with which we can estimate the state of system and reconstruct fault by designing descriptor sliding mode observer, the paper introduces an auxiliary descriptor state vector composed by a system state vector, actuator fault vector, and sensor fault vector. By the optimized method of LMI, the conditions for stability that estimated error dynamics are set up to promote the determination of the parameters designed. With this estimation, and designing a fault-tolerant controller, the system's stability can be maintained. The effectiveness of the design strategy is verified by implementing the controller in the National Renewable Energy Laboratory's 5-MW nonlinear, high-fidelity wind turbine model (FAST) and simulating it in MATLAB/Simulink.
\end{abstract}

\section{Introduction}

With the increasing demand for energy, the importance of new energy development increases with each passing day. Wind energy is an important part of new energy, and actively developing wind energy to improve the energy system structure, ease the energy crisis, and protect the environment is of great significance $[1,2]$. For engineering systems, such as wind power systems, their working conditions are poor, and, in the case of long-term operation, a failure of a subsystem or actuator or sensor failure is usually unavoidable. In most cases, we cannot predict the time of system failure. Such a failure may cause the structure and performance of the whole system to change slowly or drastically, which will seriously affect the safety of the wind turbine and its stable power output. Fault-tolerant control can guarantee the stable operation of a system under a specified performance index in the event of system component failure, thus opening a new way to improve the reliability of wind turbines in complex and harsh conditions.

In recent years, the application of model-based faulttolerant control in wind turbines has gradually expanded. Sloth et al. designed an active and passive fault-tolerant controller based on the linear variable parameter (LPV) for the pitch fault. The optimization method for the linear matrix inequality was applied to active fault-tolerant control, and the bilinear matrix inequality method was applied to passive fault-tolerant control [3]. Sami and Patton designed a sliding mode fault-tolerant control based on gain adaptive control for a $5 \mathrm{MW}$ wind turbine operating at low wind speeds. A robust observer was used to estimate the state and unknown output (sensor fault and noise), which guaranteed 
the robustness of the sliding mode to unknown output [4]. Badihi et al. designed two fault-tolerant control methods for the model uncertainties and torque actuator faults of wind turbine generator converters. One method was based on fuzzy model reference adaptive control and used fuzzy inference for parameter adaptation without prior information about any faults. The other method involved fuzzy modeling and identification technology to design a method based on the model detection and diagnosis, thus automatically using real-time fault information to correct fault signs and achieving fault-tolerant control for the torque actuator [5]. A fault-tolerant control strategy based on the unknown input observer array was proposed by Odgaard and Stoustrup. The fault detection and isolation of high-speed and low speed shaft speed sensors can be realized by an unknown input observer [6]. Blesa et al. proposed a method based on the interval observer to detect and isolate the fault of a wind turbine sensor or actuator; according to the fault information obtained, the fault-tolerant control method for the corresponding faults can be realized by using a virtual sensor or actuator technology [7].

However, the current fault-tolerant control strategies generally aim at only a single fault. Thus when faults appear in both wind turbine actuator and the sensor at the same time, guaranteeing the stable operation of the system in the specified performance index becomes challenging.

To solve the above problems, this paper proposes a fault-tolerant control strategy for the pitch system for a wind turbine based on multiple fault reconstruction. The proposed fault-tolerant control strategy consists of two parts. The first part adopts the active disturbance rejection control technology to ensure the stable output of the wind turbine power in the case of no fault. In the second part, a descriptor sliding mode observer is designed to realize the continuous estimation of the original system state, pitch actuator failure, and pitch sensor fault, and a fault-tolerant controller is designed based on the state estimation and fault estimation information to maintain the stable operation of the system under simultaneous pitch actuator and pitch sensor faults.

The remainder of this paper is organized as follows. Wind turbine modeling is presented in Section 2. A descriptor sliding mode observer is developed in Section 3. An active disturbance rejection controller used for free-faults and a reconfigurable controller used to compensate the pitch system fault effect are developed in Section 4. The proposed FFTC design is then verified in National Renewable Energy Laboratory's 5-MW nonlinear, high-fidelity wind turbine model and simulated it in MATLAB/Simulink in Section 5. The conclusions are given in Section 6.

\section{Wind Turbine Modeling}

A wind turbine system is characterized by nonlinear aerodynamic behavior and the dependence on a stochastic uncontrollable wind force as a driving signal. To conceptualize the system from the analytical and control design requirement standing points, an overall model of the turbines is required.
In this section, a generalized nonlinear wind turbine model, its pitch system, and its faults model are presented.

2.1. Nonlinear Wind Turbine Modeling. The model is shown in the form as follows [8] with a generalized nonlinear property:

$$
\dot{X}=F(X)+B u=\left[\begin{array}{l}
f_{1} \\
f_{2} \\
f_{3} \\
f_{4}
\end{array}\right]+\left[\begin{array}{l}
0 \\
0 \\
0 \\
g_{4}
\end{array}\right] u \text {. }
$$

The state vector $X$, control input $u$, and nonlinear vector $F(X)$ are defined as

$$
\begin{aligned}
X & =\left[\omega_{r}, \omega_{g}, \delta, \beta\right]^{\mathrm{T}}, \\
u & =\beta_{r} \\
F(X) & =\left[\begin{array}{cc}
\frac{P_{r}\left(x_{1}, x_{4}\right)}{x_{1} J_{r}}-\frac{x_{1} D_{s}}{J_{r}}+\frac{x_{2} D_{s}}{N_{g} J_{r}}-\frac{x_{3} K_{s}}{J_{r}} \\
\frac{x_{1} D_{s}}{N_{g} J_{g}}-\frac{x_{2} D_{s}}{N_{g}^{2} J_{g}}+\frac{x_{3} K_{s}}{N_{g} J_{g}}-\frac{T_{g}}{J_{g}} \\
x_{1}-\frac{x_{2}}{N_{g}} \\
-\frac{1}{\tau_{\beta}} x_{4}
\end{array}\right] \\
B & =\left[\begin{array}{c}
0,0,0, \frac{1}{\tau_{\beta}}
\end{array}\right]^{\mathrm{T}},
\end{aligned}
$$

where $\omega_{r}$ is the speed of low speed shaft; $\omega_{g}$ is the generator speed; $\delta$ is the twist angle; $\beta$ is the pitch angle; $\beta_{r}$ is the pitch angle control; $\tau_{\beta}$ is the time constant of the pitch actuator; $T_{g}$ is the generator torque; $J_{r}$ and $J_{g}$ are the low speed shaft and generator inertia; $N_{g}$ is the gear ratio; and $D_{s}$ and $K_{s}$ are the drive-train damping and spring constants, respectively.

The mechanical power captured by the wind turbine is described as

$$
P_{r}=\frac{1}{2} \pi \rho R^{2} V^{3} C_{p}\left(x_{1}, x_{4}, V\right),
$$

where $R$ is the rotor radius; $\rho$ is the air density; $V$ is the wind speed; and $C_{p}$ is the power conversion coefficient of the wind turbine and is a nonlinear function.

2.2. Pitch System Modeling. The system of hydraulic pitch is modeled as a closed-loop transfer function. Essentially, these position servo systems can be modeled quite well via describing a second-order transfer function as follows [9]:

$$
\frac{\beta(s)}{\beta_{r}(s)}=\frac{\omega_{n}^{2}}{s^{2}+2 \varepsilon \omega_{n} s+\omega_{n}^{2}},
$$

where $\omega_{n}$ and $\varepsilon$ are the frequency and damping ratio parameters, respectively; $\beta(s)$ is the pitch angle; and $\beta_{r}(s)$ is the pitch angle control. 
To facilitate the subsequent controller design, the state space model of the pitch system is as follows:

$$
\begin{aligned}
{\left[\begin{array}{c}
\dot{\beta} \\
\ddot{\beta}
\end{array}\right] } & =\left[\begin{array}{cc}
0 & 1 \\
-\omega_{n}^{2} & -2 \varepsilon \omega_{n}
\end{array}\right]\left[\begin{array}{c}
\beta \\
\dot{\beta}
\end{array}\right]+\left[\begin{array}{c}
0 \\
\omega_{n}^{2}
\end{array}\right] \beta_{r} \\
y & =\left[\begin{array}{ll}
1 & 0 \\
0 & 1
\end{array}\right]\left[\begin{array}{l}
\beta \\
\dot{\beta}
\end{array}\right] .
\end{aligned}
$$

2.3. Pitch Actuator Fault Modeling. The fault considered for the pitch actuator is hydraulic leakage. The dynamics of the pitch systems will be changed by a drop of oil pressure. The pressure level is modeled as a convex combination of the vertices of the parameters $\omega_{n}$ and $\varepsilon$. Hence, the pitch system can be described according to the so-called fault effectiveness parameter $\theta_{f} \in\left[\begin{array}{ll}0 & 1\end{array}\right]$, where $\theta_{f}=0$ corresponds to a faultfree actuator with $\omega_{n}=\omega_{n 0}$ and $\varepsilon=\varepsilon_{0} \theta_{f}=1$ relates to a full fault on the actuator with $\omega_{n}=\omega_{n f}, \varepsilon=\varepsilon_{f}$. Hence, the corresponding damping ratio and damping frequency can be obtained by inverse formula and $\omega_{n}$ and $\varepsilon$ can be depicted by the pitch actuator fault $[10,11]$ :

$$
\begin{gathered}
\omega_{n}^{2}=\left(1-\theta_{f}\right) \omega_{n 0}^{2}+\theta_{f} \omega_{n f}^{2} \\
\varepsilon \omega_{n}=\left(1-\theta_{f}\right) \varepsilon_{0} \omega_{n 0}+\theta_{f} \varepsilon_{f} \omega_{n f} .
\end{gathered}
$$

The corresponding state space model is as follows:

$$
\left[\begin{array}{l}
\dot{\beta} \\
\ddot{\beta}
\end{array}\right]=\left[\begin{array}{cc}
0 & 1 \\
-\omega_{n 0}^{2} & -2 \varepsilon_{0} \omega_{n 0}
\end{array}\right]\left[\begin{array}{c}
\beta \\
\dot{\beta}
\end{array}\right]+\left[\begin{array}{c}
0 \\
\omega_{n 0}^{2}
\end{array}\right] \beta_{r}+B_{a} f_{a},
$$

where $B_{\alpha}=\left[\begin{array}{c}0 \\ \omega_{n 0}^{2}\end{array}\right], f_{\alpha}=\left(1-\omega_{n f}^{2} / \omega_{n 0}^{2}\right)\left(\beta-\beta_{r}\right) \theta_{f}+2\left(\varepsilon_{0} / \omega_{n 0}-\right.$ $\left.\varepsilon_{f} \omega_{n f} / \omega_{n 0}^{2}\right) \dot{\beta} \theta_{f}$.

Equation (9) simplifies the design of the observer. However, $f_{\alpha}$ is the newly defined actuator fault signal and it may not be able to sufficiently reflect the severity of the primordial fault $\theta_{f}$ and it is a potential problem. One method to refind the primordial fault signal is to put (10) into use:

$$
\begin{aligned}
& \theta_{f} \\
& =\frac{f_{\alpha}}{\left(1-\omega_{n f}^{2} / \omega_{n 0}^{2}\right)\left(\beta-\beta_{r}\right)+2\left(\varepsilon_{0} / \omega_{n 0}-\varepsilon_{f} \omega_{n f} / \omega_{n 0}^{2}\right) \dot{\beta}} .
\end{aligned}
$$

2.4. Pitch Sensor Faults Modeling. There are three common faults for the pitch sensor: biased output, fixed output, and no output. The only difference between the front two faults original from the pitch sensor is that detecting a fixed output is necessary $[12,13]$.

2.4.1. Biased Output on Pitch Sensor. The closed-loop pitch system and the pitch angle measurement are affected by a biased pitch sensor measurement. While the bias occurs, the pitch sensor fault model is as follows:

$$
\beta_{\text {mes }}(t)=\beta(t)+\beta_{\text {bias }}(t),
$$

where $\beta_{\text {bias }}(t)$ is the pitch angle measurement bias and $\beta(t)$ is the actual pitch angle.
2.4.2. Fixed Output on Pitch Sensor. A fixed output on a pitch sensor is an abrupt fault and can occur at any time leading to the following measurement equation:

$$
\beta_{\text {mes }}(t)=\beta\left(t_{f}\right), \quad \forall t>t_{f},
$$

where $t_{f}$ is the time when the fault occurs.

2.4.3. No Output on Pitch Sensor. No output on a pitch sensor causes the same changes in the measurement equation as a fixed output. Contrary to a fixed output, the control system is informed when there is no output from the pitch sensor. The fault model is as follows:

$$
\beta_{\text {mes }}(t)=0, \quad \forall t>t_{f} .
$$

\section{Descriptor Sliding Mode Observer Design}

3.1. Problem Description. Considering the following wind turbine pitch system with four conditions, pitch actuator fault, pitch sensor fault, system uncertainty, and external disturbance [14-17], the corresponding dynamic equations are as follows:

$$
\begin{aligned}
& \dot{x}(t)=A x(t)+B u(t)+B_{a} f_{a}(t)+M d(t, x, u) \\
& y(t)=C x(t)+D_{s} f_{s}(t)+D_{a} f_{a}(t),
\end{aligned}
$$

where $x(t) \in \mathbb{R}^{n}$ is the system state vector; $y(t) \in \mathbb{R}^{p}$ is the measurement output vector; $u(t) \in \mathbb{R}^{m}$ is the vector of control input; $d(t, x, u) \in \mathbb{R}^{h}$ is external and uncertainty disturbance; $f_{a}(t) \in \mathbb{R}^{q}$ is the vector of pitch actuator fault; $f_{s}(t) \in \mathbb{R}^{l}$ is the vector of pitch sensor fault; and $A \in \mathbb{R}^{n \times n}, B \in \mathbb{R}^{n \times m}$, $B_{a} \in \mathbb{R}^{n \times q}, M \in \mathbb{R}^{n \times h}, C \in \mathbb{R}^{p \times n}$, and $D_{s} \in \mathbb{R}^{p \times l}, D_{a} \in \mathbb{R}^{p \times q}$, are known constant matrices.

Some assumptions are made in this paper for subsequent theoretical analyses and controller design.

Assumption 1. The conditions for the uncertainty and external disturbance are bounded as the following norm, and pitch actuator fault and pitch sensor fault are held:

$$
\begin{aligned}
\|d(\cdot)\| & \leq d_{0}, \\
\left\|f_{a}(t)\right\| & \leq \alpha_{0}, \\
\left\|\dot{f}_{a}(t)\right\| & \leq \alpha_{1}, \\
\left\|f_{s}(t)\right\| & \leq \beta_{0},
\end{aligned}
$$

where $d_{0}>0, \alpha_{0}>0, \alpha_{1}>0$, and $\beta_{0}>0$ are some known constants.

Assumption 2. The pair $(A, B)$ is stable, and $(A, C)$ is tested. And a constant $\delta>0$ exits such that

$$
\operatorname{rank}\left[\begin{array}{cc}
\delta I_{n}+A & B_{a} \\
C & 0
\end{array}\right]=n+q .
$$

Assumption 3. The matrices $M$ and $D_{s}$ are full column rank, and the dimension standard of $q+l \leq p$ holds. 
The purpose of this paper is to get the estimates of pitch system state and fault simultaneously. For the objective, we can define the new augmented variables and matrices as follows:

$$
\begin{aligned}
& x_{s}(t) \triangleq D_{s} f_{s}(t) \text {, } \\
& \bar{x}(t) \triangleq\left[\begin{array}{c}
x(t) \\
f_{a}(t) \\
x_{s}(t)
\end{array}\right], \\
& \bar{A} \triangleq\left[\begin{array}{ccc}
A & 0 & 0 \\
0 & -\delta I_{q} & 0 \\
0 & 0 & -I_{p}
\end{array}\right], \\
& \bar{B} \triangleq\left[\begin{array}{c}
B \\
0_{q \times m} \\
0_{p \times m}
\end{array}\right] \text {, } \\
& \bar{C} \triangleq\left[\begin{array}{lll}
C & 0_{p \times q} & I_{p}
\end{array}\right] \text {, } \\
& \bar{E} \triangleq\left[\begin{array}{ccc}
I_{n} & \delta^{-1} B_{a} & 0 \\
0 & I_{q} & 0 \\
0 & 0 & 0_{p \times p}
\end{array}\right], \\
& \bar{N} \triangleq\left[\begin{array}{c}
0_{n \times p} \\
0_{q \times p} \\
I_{p}
\end{array}\right] \text {, } \\
& \bar{B}_{f} \triangleq\left[\begin{array}{ccc}
\delta^{-1} B_{a} & 0_{n \times l} & M \\
I_{q} & 0_{q \times l} & 0_{q \times h} \\
0_{p \times q} & D_{s} & 0_{p \times h}
\end{array}\right] \text {, } \\
& \bar{f}(t) \triangleq\left[\begin{array}{c}
\delta f_{a}(t)+\dot{f}_{a}(t) \\
f_{s}(t) \\
d(\cdot)
\end{array}\right], \\
& \bar{n} \triangleq n+q+p,
\end{aligned}
$$

where $\delta>0$ is a design parameter selected such that Assumption 2 holds.

By the above definitions, we can construct a new descriptor system equivalently as follows:

$$
\begin{aligned}
\bar{E} \dot{\bar{x}}(t) & =\bar{A} \bar{x}(t)+\bar{B} u(t)+\bar{B}_{f} \bar{f}(t) \\
y(t) & =\bar{C} \bar{x}(t),
\end{aligned}
$$

where $\bar{x}(t)$ is the descriptor system state, composed of the initial state, the pitch actuator fault, and the pitch sensor fault, which is imported into equivalently establishing a system for descriptor (18).
3.2. Descriptor Sliding Mode Observer Design. The descriptor observer can be designed by the lemma as the following shows, by the summaries from [18].

Lemma 4. There always exists a matrix $\bar{L}_{D} \in \mathbb{R}^{\bar{n} \times p}$ designed such that $\bar{S} \triangleq\left(\bar{E}+\bar{L}_{D} \bar{C}\right)$ is nonsingular; that is, the matrix $\bar{W} \triangleq \bar{S}^{-1}$ exists. Furthermore, it holds that $\bar{C} \bar{W} \bar{L}_{D}=I_{p}$ and $\bar{A} \bar{W} \bar{L}_{D}=-\bar{N}$.

On the basis of Lemma 4, we can design the descriptor sliding mode observer for the descriptor plant (18) as follows:

$$
\begin{aligned}
\bar{S} \dot{\xi}(t) & =\left(\bar{A}-\bar{L}_{P} \bar{C}\right) \xi(t)-\bar{N} y(t)+\bar{B} u(t)+\bar{L}_{s} u_{s}(t) \\
\widehat{\bar{x}}(t) & =\xi(t)+\bar{W} \bar{L}_{D} y(t),
\end{aligned}
$$

where $\xi(t) \in \mathbb{R}^{\bar{n}}$ is a variable in the middle, $\hat{\bar{x}}(t) \in \mathbb{R}^{\bar{n}}$ is the estimator of descriptor system state, $\bar{L}_{P} \in \mathbb{R}^{\bar{n} \times p}, \bar{L}_{D} \in \mathbb{R}^{\bar{n} \times p}$, and $\bar{L}_{s} \in \mathbb{R}^{\bar{n} \times(q+l+h)}$ are the derivative gain, proportion gain, and sliding mode gain, and $u_{s}(t) \in \mathbb{R}^{(q+l+h)}$ is the input of discontinuous sliding mode to get rid of the disturbances of system faults and uncertainties.

We can define the design matrix $\bar{L}_{D}$ as follows:

$$
\bar{L}_{D}=\left[\begin{array}{l}
\bar{L}_{D 1} \\
\bar{L}_{D 2} \\
\bar{L}_{D 3}
\end{array}\right]=\left[\begin{array}{c}
0_{n \times p} \\
0_{q \times p} \\
Z
\end{array}\right] \text {, }
$$

where $Z=z I_{p}$ and $z>0$ is a design scalar.

Lemma 5. If Assumption 2 holds, a proportional gain $\bar{L}_{P}=$ $\bar{S} \bar{X}^{-1} \bar{C}^{T}$ will exist, such that the matrix $\bar{W}\left(\bar{A}-\bar{L}_{P} \bar{C}\right)$ is Hurwitz, where we can solve $\bar{X}>0$ via the Lyapunov equation:

$$
-(\mu I+\bar{W} \bar{A})^{T} \bar{X}-\bar{X}(\mu I+\bar{W} \bar{A})=-\bar{C}^{T} \bar{C},
$$

where $\mu>0$ satisfies $\operatorname{Re}\left[\lambda_{i}(\bar{W} \bar{A})\right]>-\mu, \forall i \in\{1,2, \ldots, \bar{n}\}$.

The final step is to form the input term $u_{s}(t)$ of sliding mode to make the input disturbance $\bar{f}(t)$ attenuated. At first, the gain matrix $\bar{L}_{s}(t)$ for sliding mode can be designed as

$$
\bar{L}_{s}=\bar{B}_{f} .
$$

Then, we can define the surface of sliding mode with $\widetilde{s}(t) \in \mathbb{R}^{(q+l+h)}$ as follows:

$$
\widetilde{s}(t)=\bar{B}_{f}^{T} \bar{W}^{T} \bar{P} \bar{e}(t),
$$

where the descriptor error is $\bar{e}(t)=\widehat{\bar{x}}(t)-\bar{x}(t)$ and the Lyapunov matrix $\bar{P}>0$ is designed such that the following constraint holds:

$$
\bar{B}_{f}^{T} \bar{W}^{T} \bar{P}=\bar{H} \bar{C}
$$

where $\bar{H} \in \mathbb{R}^{(q+l+h) \times p}$ is the matrix of design parameter based on matrix $\bar{P}$. 
Remark 6. Constraint (24) is introduced because $\bar{e}(t)$ is unknown in the function (23) of sliding mode; thus, $\widetilde{s}(t)$ cannot be got. Note that $\bar{C} \bar{e}(t)=\bar{C} \overline{\bar{x}}(t)-\bar{C} \bar{x}(t)$ is available, because $\bar{C} \bar{x}(t)$ and $\bar{C} \bar{x}(t)$ can be obtained. Therefore, on the basis of the constraint (24), we can derive it as follows:

$$
\widetilde{s}(t)=\bar{H} \bar{C} \bar{e}(t) .
$$

It is presented that $\widetilde{\mathcal{s}}(t)$ is available to design the observer. At present, the discontinuous sliding mode input term $u_{s}(t)$ can be designed in the form as the following shows:

$$
u_{s}(t)=-\gamma_{1} \operatorname{sgn}(\widetilde{s}(t)),
$$

where $\gamma_{1}>0$ is a small designed value.

3.3. The Descriptor Error and Estimation State Dynamics. First, we can rewrite the observer (19) as follows:

$$
\begin{aligned}
\bar{S} \dot{\bar{x}}(t)= & \bar{S} \dot{\xi}(t)+\bar{L}_{D} \dot{y}(t) \\
= & \left(\bar{A}-\bar{L}_{P} \bar{C}\right)\left(\hat{\bar{x}}(t)-\bar{W} \bar{L}_{D} y(t)\right)-\bar{N} y(t) \\
& +\bar{B} u(t)+\bar{L}_{s} u_{s}(t)+\bar{L}_{D} \dot{y}(t) \\
= & \left(\bar{A}-\bar{L}_{P} \bar{C}\right) \hat{\bar{x}}(t)-\bar{A} \bar{W} \bar{L}_{D} y(t) \\
& +\bar{L}_{P} \bar{C} \bar{W} \bar{L}_{D} \bar{C} \bar{x}-\bar{N} y(t)+\bar{B} u(t)+\bar{L}_{s} u_{s}(t) \\
& +\bar{L}_{D} \bar{C} \dot{\bar{x}}(t) .
\end{aligned}
$$

Using Lemma 4, (27) is simplified to

$$
\begin{aligned}
\bar{S} \dot{\bar{x}}(t)= & \left(\bar{A}-\bar{L}_{P} \bar{C}\right) \hat{\bar{x}}(t)+\bar{L}_{P} \bar{C} \bar{x}+\bar{B} u(t)+\bar{L}_{s} u_{s}(t) \\
& +\bar{L}_{D} \bar{C} \dot{\bar{x}}(t) .
\end{aligned}
$$

Adding $\bar{L}_{D} \bar{C} \dot{\bar{x}}(t)$ to both sides of the descriptor system (18), it can be rewritten as

$$
\begin{aligned}
\bar{S} \dot{\bar{x}}(t)= & \left(\bar{A}-\bar{L}_{P} \bar{C}\right) \bar{x}(t)+\bar{L}_{P} \bar{C} \bar{x}(t)+\bar{B} u(t) \\
& +\bar{B}_{f} \bar{f}(t)+\bar{L}_{D} \bar{C} \dot{\bar{x}}(t) .
\end{aligned}
$$

Subtracting (29) from (28), the descriptor error dynamics becomes

$$
\dot{\bar{e}}(t)=\bar{W}\left(\bar{A}-\bar{L}_{P} \bar{C}\right) \bar{e}(t)+\bar{W}\left(\bar{L}_{s} u_{s}(t)-\bar{B}_{f} \bar{f}(t)\right) .
$$

Then, we can derive the system equation of sliding mode estimation state. First, we can rewrite the observer state Eq. (28) as follows:

$$
\begin{aligned}
\overline{\bar{E} \dot{\bar{x}}}(t)+\bar{L}_{D} \overline{\bar{C}} \dot{\bar{x}}(t)= & \bar{A} \hat{\bar{x}}(t)-\bar{L}_{P} \bar{C} \bar{e}(t)+\bar{B} u(t) \\
& +\bar{L}_{s} u_{s}(t)+\bar{L}_{D} \bar{C} \dot{\bar{x}}(t)
\end{aligned}
$$

which is equivalent to

$$
\begin{aligned}
\bar{E} \dot{\bar{x}}(t)= & \bar{A} \hat{\bar{x}}(t)-\bar{L}_{P} \bar{C} \bar{e}(t)+\bar{B} u(t)+\bar{L}_{s} u_{s}(t) \\
& -\bar{L}_{D} \bar{C} \dot{\bar{e}}(t) .
\end{aligned}
$$

We can decompose the matrices $\bar{L}_{P}, \bar{L}_{s}$, and $u_{s}(t)$ as follows:

$$
\begin{gathered}
\bar{L}_{P} \triangleq\left[\begin{array}{l}
L_{P 1} \\
L_{P 2} \\
L_{P 3}
\end{array}\right], \\
\bar{L}_{s} \triangleq\left[\begin{array}{l}
L_{s 1} \\
L_{s 2} \\
L_{s 3}
\end{array}\right], \\
u_{s}(t) \triangleq\left[\begin{array}{l}
u_{s 1}(t) \\
u_{s 2}(t) \\
u_{s 3}(t)
\end{array}\right] .
\end{gathered}
$$

From $\bar{L}_{s}=\bar{B}_{f}$, it can be easily obtained that

$$
L_{s 1}=\left[\begin{array}{lll}
\delta^{-1} B_{a} & 0_{n \times l} & M
\end{array}\right]
$$

and from (20), we can derive it as

$$
\bar{L}_{D} \bar{C} \dot{\bar{e}}(t)=\left[\begin{array}{c}
0_{n \times p} \\
0_{q \times p} \\
z \bar{C} \dot{\bar{e}}(t)
\end{array}\right] .
$$

Subsequently, the state Eq. (32) is decomposed to obtain the following estimation state Eq. (36):

$$
\begin{aligned}
\dot{\hat{x}}(t)= & A \widehat{x}(t)-L_{P 1} \bar{C} \bar{e}(t)+B u(t)+\delta^{-1} B_{a} u_{s 1}(t) \\
& +M u_{s 3}(t)-\delta^{-1} B_{a} \dot{\hat{f}}_{a}(t) .
\end{aligned}
$$

Second, an estimation state is constructed based on the integral sliding mode surface $\widehat{x}(t)$ as follows:

$$
\widehat{\mathcal{s}}(t)=G \widehat{x}(t)-\int_{0}^{t} G(A+B K) \widehat{x}(\tau) d \tau,
$$

where $G \in \mathbb{R}^{m \times n}$ and $K \in \mathbb{R}^{m \times n}$ are the matrices with parameter designed. $G$ is chosen such that $G B$ is nonsingular, and $K$ is designed such that $A+B K$ is Hurwitz. From (36) and (37), it yields

$$
\begin{aligned}
\dot{\dot{s}}(t) & =G\left(-L_{P 1} \bar{C} \bar{e}(t)+\delta^{-1} B_{a} u_{s 1}(t)+M u_{s 3}(t)\right. \\
- & \left.\delta^{-1} B_{a} \dot{\hat{f}}_{a}(t)\right)+G B(u(t)-K \hat{x}(t)) .
\end{aligned}
$$

Assuming that there exist matrices $B_{1} \in \mathbb{R}^{m \times q}$ and $B_{2} \in$ $\mathbb{R}^{m \times h}$ such that $B_{a}=B B_{1}$ and $M=B B_{2}$, and letting $\dot{\widehat{s}}(t)=0$, we can derive the equivalent control scheme as follows:

$$
\begin{aligned}
u_{\mathrm{eq}}(t)= & K \widehat{x}(t)+\delta^{-1} B_{1}\left(\dot{\hat{f}}_{a}(t)-u_{s 1}(t)\right)-B_{2} u_{s 3}(t) \\
& +(G B)^{-1} G L_{P 1} \bar{C} \bar{e}(t) .
\end{aligned}
$$


Substituting (39) into (36), we can obtain the state system equation with the method of sliding mode estimation as the following shows:

$$
\begin{aligned}
\dot{\hat{x}}(t)= & (A+B K) \widehat{x}(t) \\
& +\left(B(G B)^{-1} G-I_{n}\right) L_{P 1} \bar{C} \bar{e}(t) .
\end{aligned}
$$

Combine the sliding mode estimation state Eq. (40) with the descriptor error system (25) and build a system with the closed-loop property as (41) shows:

$$
\begin{aligned}
& \dot{\hat{x}}(t)=(A+K B) \widehat{x}(t)+\left(B(G B)^{-1} G-I_{n}\right) L_{P 1} \bar{C} \bar{e}(t) \\
& \dot{\bar{e}}(t)=\bar{W}\left(\bar{A}-\bar{L}_{P} \bar{C}\right) \bar{e}(t)+\bar{W}\left(\bar{L}_{s} u_{s}(t)-\bar{B}_{f} \bar{f}(t)\right) .
\end{aligned}
$$

\subsection{The Stability Condition of Observer}

Theorem 7. Based on the sliding mode input scheme as $u_{s}(t)$ (26) presented, the system shown in (41) is stable, if there exist $R \in \mathbb{R}^{n \times n}>0, \bar{P} \in \mathbb{R}^{\bar{n} \times \bar{n}}>0$, and $\bar{H} \in \mathbb{R}^{(q+l+h) \times p}$ such that the constraint (24) holds and the LMI optimization problem admits a feasible solution [14]:

$\min (\theta+\varepsilon)$,

$$
\text { s.t. } \quad \Psi=\left[\begin{array}{cccccc}
\Psi_{11} & \Psi_{12} & \Psi_{13} & \Psi_{14} & 0 & 0 \\
\Psi_{12}^{T} & \Psi_{22} & 0 & 0 & \Psi_{25} & \Psi_{26} \\
\Psi_{13}^{T} & 0 & \Psi_{33} & 0 & 0 & 0 \\
\Psi_{14}^{T} & 0 & 0 & \Psi_{44} & 0 & 0 \\
0 & \Psi_{25}^{T} & 0 & 0 & \Psi_{55} & 0 \\
0 & \Psi_{26}^{T} & 0 & 0 & 0 & \Psi_{66}
\end{array}\right]<0 \text {, }
$$

where $\Psi_{11}=(A+B K)^{T} R+(A+B K), \Psi_{12}=R\left(B(G B)^{-1} G-\right.$ $\left.I_{n}\right) L_{P 1} \bar{C}, \Psi_{13}=I_{n}, \Psi_{25}=I_{\bar{n}}, \Psi_{14}=R\left(I_{n}-B(G B)^{-1} G\right), \Psi_{26}=$ $\bar{P} \bar{W}, \Psi_{22}=\left(\bar{A}-\bar{L}_{P} \bar{C}\right)^{T} \bar{W}^{T} \bar{P}+\bar{P} \bar{W}\left(\bar{A}-\bar{L}_{P} \bar{C}\right), \Psi_{33}=-\theta I_{n}$, $\Psi_{44}=-\varepsilon I_{n}, \Psi_{55}=-\theta I_{\bar{n}}, \Psi_{66}=-\varepsilon I_{\bar{n}}$.

\section{Active Fault-Tolerant Control Design}

The fault-tolerant control of a wind turbine in area 3 (above rated wind speed) is mainly studied in this paper. The output power of the generator must be adjusted to the rated power to ensure the output quality and wind turbine safety. There are two general methods for power regulation [19]: (1) With the generator torque fixed and the generator speed adjusted to the rated speed by controlling the pitch angle and (2) the generator torque and pitch angle are adjusted simultaneously. However, regardless of which method is used, the pitch system must be used to regulate the pitch angle. Therefore, the pitch actuator and pitch sensor are very important parts of the power control system. The purpose of this paper to develop a novel active fault-tolerant controller to address the case in which the pitch actuator and pitch sensor show faults at the same time, so only a collective pitch method is used. Method (1) will be chosen for the power control in this paper.
An active fault-tolerant control for the pitch system (14) comprises a norm controller and a reconfigurable controller, designed as

$$
u(t)=u_{\mathrm{norm}}+u_{f}
$$

where $u_{\text {norm }}$ is the norm controller to achieve pitch angle control under the fault-free case and $u_{f}$ is the reconfigurable controller to compensate the fault effect.

4.1. Norm Controller Design. The active disturbance rejection control (ADRC) technique is used in wind turbine pitch control under the fault-free case in this paper due to its facilitation and robustness. The ADRC controller design procedures are summarized as the following three steps [20, 21]:

(1) Firstly, to avoid a large overshoot of the system, the step input signal is transformed into a continuous and smooth signal by designing the tracking differentiator [22].

(2) Second, an extended state and disturbance nonlinear observer is designed to estimate and compensate for the unknown time varying nonlinear disturbances in the system online.

(3) Finally, the pitch control is realized by a conventional PD controller.

It is assumed that the output of the system is the speed of the low speed shaft and that the input is the pitch angle. From (1) to (3), the dynamic equation of low speed shaft speed is

$$
\dot{x}_{1}=\frac{P_{r}\left(x_{1}, x_{4}\right)}{x_{1} J_{r}}-\frac{x_{1} D_{s}}{J_{r}}+\frac{x_{2} D_{s}}{N_{g} J_{r}}-\frac{x_{3} K_{s}}{J_{r}} .
$$

Its second-order derivative can be obtained as

$$
\begin{aligned}
\frac{d^{2} x_{1}}{d t^{2}} & =L_{f}(x)+L_{g}(x) u \\
L_{f}(x) & =\sum_{i=1}^{4}\left(\frac{\partial f_{1}}{\partial x_{i}} f_{i}\right)+\frac{\partial f_{1}}{\partial V} \dot{V} \\
L_{g}(x) & =\frac{\partial f_{1}}{\partial x_{4}} g_{4},
\end{aligned}
$$

where $\dot{V}$ is the derivative of the wind speed.

Assuming that all nonlinearities of the system represented as $L_{f}(x)$ and $L_{g}(x)$ in (45) are unknown, the defined perturbation that contains all nonlinear and time varying dynamics of the system is shown as follows:

$$
\psi(x)=L_{f}(x)+\left(L_{g}(x)-b_{0}\right) u,
$$

where $b_{0}=L_{g}(x)_{x=x(0)}$ is the nominal constant gain.

Then, system (45) can be represented as follows:

$$
\frac{d^{2} x_{1}}{d t^{2}}=\psi(x)+b_{0} u
$$


4.1.1. Design of Nonlinear State and Perturbation Observer. Defining $z_{1}=x_{1}, z_{2}=\dot{x}_{1}$, and $z_{3}=\psi(x)$, the mathematical model of the observer is shown as follows:

$$
\begin{aligned}
& \dot{z}_{1}=z_{2} \\
& \dot{z}_{2}=z_{3}+b_{0} u \\
& \dot{z}_{3}=\dot{\psi}(x) .
\end{aligned}
$$

Defining $\widetilde{z}_{i}=z_{i}-\widehat{z}_{i}$, the nonlinear observer is then shown as follows:

$$
\begin{aligned}
\dot{\vec{z}}_{1} & =\widehat{z}_{2}+k_{01} \widetilde{z}_{1} \\
\dot{\vec{z}}_{2} & =\widehat{z}_{3}+b_{0} u+k_{02} f a l\left(\widetilde{z}_{1}, 0.5, h\right) \\
\dot{\vec{z}}_{3} & =k_{03} \text { fal }\left(\widetilde{z}_{1}, 0.25, h\right) \\
\operatorname{fal}(\chi, \sigma, h) & = \begin{cases}\frac{\sigma^{2}}{h^{(1-\sigma)}} \chi, & |\chi| \leq h \\
\operatorname{sign}(\chi) \sigma^{2}|\chi|^{\delta}, & |\chi|>h,\end{cases}
\end{aligned}
$$

where $\widehat{z}_{i}$ is the estimated value of $z_{i} ; k_{0 i}$ is a gain of the observer; $\chi$ is an input error of the nonlinear function; $0<$ $\sigma<1$ is the precision index; and $h$ is the width of the linear area of a nonlinear function.

4.1.2. The Pitch Controller Design. In this controller, the actual perturbation is compensated in real-time by estimating the unknown perturbation of the system with a nonlinear observer. The pitch controller can be expressed as

$$
u_{\text {norm }}=\frac{1}{b_{0}}\left(K_{P}\left(v_{1}-\widehat{z}_{1}\right)+K_{D}\left(v_{2}-\widehat{z}_{2}\right)-\widehat{z}_{3}\right) \text {, }
$$

where $K_{P}$ and $K_{D}$ are two control gains; $\widehat{z}_{1}, \widehat{z}_{2}$, and $\widehat{z}_{3}$ are the outputs of the observer; and $v_{1}, v_{2}$ are the outputs of the tracking differentiator.

4.2. Reconfigurable Controller Design. With the above-mentioned stability conditions, we can guarantee the reachability of the sliding surfaces $\widetilde{s}(t)$ of $(25)$ and $\widehat{s}(t)$ of (37). Then presenting the fault-tolerant control law as follows:

$$
\begin{aligned}
u_{f}= & K \hat{x}(t)-\delta^{-1} G B_{a} u_{s 1}(t)-G M u_{s 3}(t)-\gamma_{2} \widehat{s}(t) \\
& -\rho(t) \operatorname{sgn}(\widehat{s}(t))
\end{aligned}
$$

where $\rho(t)=\left\|G L_{P 1}\right\|\|\overline{\bar{C}} \bar{e}(t)\|+\delta^{-1}\left\|G B_{a}\right\|\left\|\dot{\hat{f}}_{a}(t)\right\|, \gamma_{2}>0$ is a small design parameter, and $G$ is designed such that $G B=I_{m}$.

Because it is not as easy to obtain a solution under limit (24) directly in MATLAB, constraint (24) can be transformed into the following inequality condition:

$$
\left(\bar{B}_{f}^{T} \bar{W}^{T} \bar{P}-\bar{H} \bar{C}\right)^{T}\left(\bar{B}_{f}^{T} \bar{W}^{T} \bar{P}-\bar{H} \bar{C}\right)<\vartheta I_{\bar{n}}
$$

where $\vartheta>0$ is a small parameter. By using the Schur complement Lemma, we can convert condition (55), equivalently, into

$$
\begin{aligned}
& \min (\vartheta) \\
& \text { s.t. } \quad \Pi=\left[\begin{array}{cc}
-\vartheta I_{\bar{n}} & \left(\bar{B}_{f}^{T} \bar{W}^{T} \bar{P}-\bar{H} \bar{C}\right)^{T} \\
\left(\bar{B}_{f}^{T} \bar{W}^{T} \bar{P}-\bar{H} \bar{C}\right) & I_{q+l+h}
\end{array}\right] \\
& <0 \text {. } \\
& \min (\omega(\theta+\varepsilon)+(1-\omega) \vartheta) \\
& \text { s.t. } \Psi<0 \text {, } \\
& \Pi<0 \text {, }
\end{aligned}
$$

Therefore, we can transform the LMI conditions of (24) and (56) into a problem of LMI optimization to get viable solutions. Recalling the minimization of $\theta$ and $\varepsilon$, the parameter $\vartheta$ must be minimized. To this end, we can obtain

where $\omega$ is a fixed parameter to be selected such that $0<$ $\omega<1$. By using the mincx solver in the standard MATLAB LMI Control Toolbox, we can solve the LMI minimization optimization problem.

With the descriptor sliding mode observer (19) proposing, we can now directly estimate the augmented descriptor system state $\bar{x}(t)$. Note that because $D_{s}$ is a full column rank, as supposed in Assumption 3, $\left(D_{s}^{T} D_{s}\right)^{-1}$ exists. Hence, the estimate of $f_{s}$ is computed as follows:

$$
\widehat{f}_{s}(t)=I_{l}\left(D_{s}^{T} D_{s}\right)^{-1} D_{s}^{T}\left[\begin{array}{lll}
0_{p \times n} & 0_{p \times q} & I_{p}
\end{array}\right] \widehat{\bar{x}}(t) .
$$

Remark 8. To prevent the signals $u_{s}(t)$ and $u(t)$ from becoming chatter, the continuous functions $\widetilde{s}(t) /(\|\widetilde{s}(t)\|+o)$ and $\widehat{s}(t) /(\|\widehat{s}(t)\|+o)$ can be simply used to replace $\operatorname{sgn}(\widetilde{s}(t))$ and $\operatorname{sgn}(\widehat{s}(t))$, where $o>0$ is a small constant [23].

We can summarize the parameters of the descriptor sliding mode method and the fault-tolerant control design process into the following four steps:

(1) Choose $\delta$ properly such that condition (16) holds, and select the derivative gain matrix $\bar{L}_{D}$ in the form (20) such that $\bar{S} \triangleq\left(\bar{E}+\bar{L}_{D} \bar{C}\right)$ is nonsingular.

(2) Choose $\mu>0$ such that $\operatorname{Re}\left[\lambda_{i}(\bar{W} \bar{A})\right]>-\mu$ for $\forall i \in\{1,2, \ldots, \bar{n}\}$. Deal with the Lyapunov Eq. (21) to obtain $\bar{X}$. Furthermore, compute the proportional gain matrix $\bar{L}_{P}$ as $\bar{L}_{P}=\bar{S} \bar{X}^{1} \bar{C}^{T}$.

(3) Design the gain matrix $\bar{L}_{s}$ of sliding mode, such that $\bar{L}_{s}=\bar{B}_{f}$. By using the mincx solver in MATLAB, choose a fixed scalar $\omega$ and have the LMI optimization problem (57) solved to get the minimized $\theta, \varepsilon$, and $\vartheta$ and the viable solutions $R, \bar{P}$, and $\bar{H}$. Design $u_{s}(t)$ as the equation of (26). 
(4) Design $G$ such that $G B=I_{m}$. Choose $K$ such that $(A+$ $B K)$ is Hurwitz. Let $L_{P 1}=\left[\begin{array}{lll}I_{n \times n} & 0_{n \times q} & 0_{n \times p}\end{array}\right] \bar{L}_{P}, u_{s 1}=$ $\left[\begin{array}{lll}I_{q \times q} & 0_{q \times l} & 0_{q \times h}\end{array}\right] u_{s}$, and $u_{s 3}=\left[\begin{array}{lll}0_{h \times q} & 0_{h \times l} & I_{h \times h}\end{array}\right] u_{s}$, and design $u(t)$ as (43).

\section{Simulation Results}

In this paper, the faults considered for the pitch system are hydraulic leakage on pitch actuator and a biased output on pitch sensor. Hence, following the design procedures mentioned above, the effective design and verification for the proposed controller can be shown via pitch system (14) with

$$
\begin{aligned}
A & =\left[\begin{array}{cc}
0 & 1 \\
-123.4321 & -13.3320
\end{array}\right], \\
B & =\left[\begin{array}{c}
0 \\
123.4321
\end{array}\right], \\
B_{a} & =\left[\begin{array}{c}
0 \\
123.4321
\end{array}\right], \\
M & =\left[\begin{array}{l}
-0.2 \\
0
\end{array}\right], \\
C & =\left[\begin{array}{ll}
1 & 0 \\
0 & 1
\end{array}\right], \\
D_{a} & =\left[\begin{array}{l}
1 \\
1
\end{array}\right], \\
D_{s} & =\left[\begin{array}{l}
1 \\
0
\end{array}\right], \\
\omega_{n 0} & =11.11 \mathrm{rad} / \mathrm{s}, \\
\omega_{n f} & =3.42 \mathrm{rad} / \mathrm{s}, \\
\varepsilon_{0} & =0.6, \\
\varepsilon_{f} & =0.9, \\
d(\cdot) & =0.2 \mathrm{cos}(6 t)+0.5 \sin (2 t) \\
\theta_{f} & = \begin{cases}0, & 0 \leq t \leq 70 \\
1, & 70<t \leq 100,\end{cases} \\
f_{s}(t) & = \begin{cases}0, \\
f_{s} \cdot \beta(t), & 40<t \leq 100 .\end{cases} \\
& =0 \leq t \leq 40 \\
&
\end{aligned}
$$

The simulations are carried out within FASTMATLAB/Simulink combined environment, and the detailed parameters of NREL's 5-MW benchmark wind turbine model are described in Table 1. We can do simulations with the wind speed time signal and its average speed is $16 \mathrm{~m} / \mathrm{s}$, as shown in Figure 1.
TABLE 1: Parameters of NREL's $5 \mathrm{MW}$ wind turbine model.

\begin{tabular}{lc}
\hline Power rating & $5 \mathrm{MW}$ \\
Rotor orientation, structure & Upwind, three-bladed \\
Control & Variable speed, variable pitch \\
Rotor, hub diameter & $126 \mathrm{~m}, 3 \mathrm{~m}$ \\
Hub height & $90 \mathrm{~m}$ \\
Cut in, rated, cut out wind speed & $3 \mathrm{~m} / \mathrm{s}, 11.4 \mathrm{~m} / \mathrm{s}, 25 \mathrm{~m} / \mathrm{s}$ \\
Rated rotor, generator speed & $12.1 \mathrm{rpm}, 1173.7 \mathrm{rpm}$ \\
Maximum blade pitch rate & $8 \mathrm{deg} / \mathrm{s}$ \\
Rated generator torque & $43093 \mathrm{Nm}$ \\
Maximum generator torque & $47402 \mathrm{Nm}$ \\
\hline
\end{tabular}

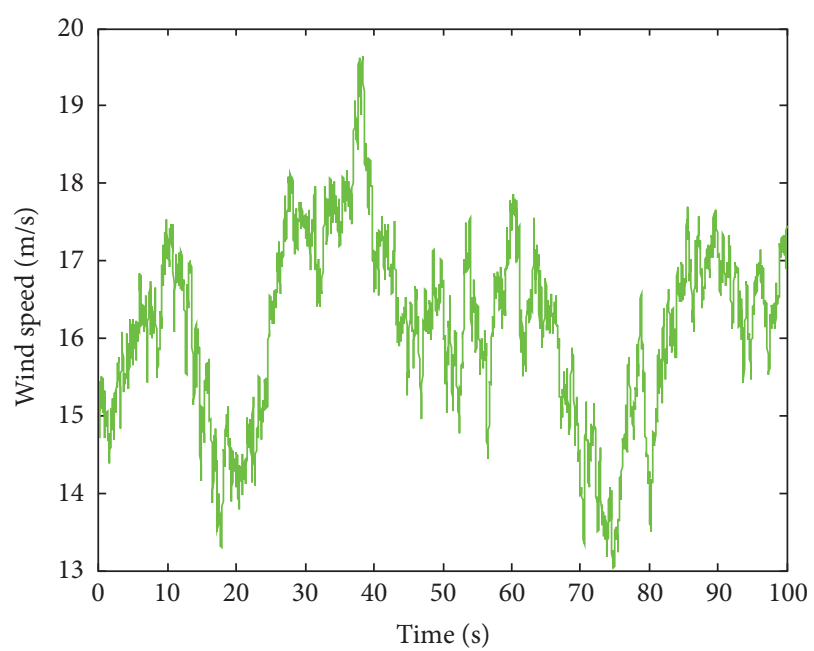

FIGURE 1: Effective wind speed.

Let the parameter $\delta=0.55$, which makes sure that condition (16) is available, and select the derivative gain max: $\bar{L}_{D}=\left[\begin{array}{l}00010 \\ 00001\end{array}\right]^{T}$; then, the matrix $\bar{S}$ can be made nonsingular, and $\bar{S}$ and $\bar{W}$ can be calculated.

We select $\mu=-\lambda_{\min }(\bar{W} \bar{A})+5=5.55$ such that $\operatorname{Re}\left[\lambda_{i}(\bar{W} \bar{A})\right]>-\mu$ for $i=1,2, \ldots, \bar{n}$. Via solving the Lyapunov equation (21), we can get the matrix $\bar{X}$ and thus also can calculate the proportional gain matrix as

$$
\begin{aligned}
\bar{L}_{P} & =10^{4} \\
& \times\left[\begin{array}{ccccc}
0.0001 & 0.0165 & -0.0001 & 0.0009 & 0.0000 \\
-0.0051 & -1.0953 & 0.0049 & 0.0000 & 0.0015
\end{array}\right]^{T} .
\end{aligned}
$$

By selecting the scalar $₫$, the LMI optimization problem (57) can be resolved and we can get the practical solutions of $R$ and $\bar{H}$ as follows:

$$
\begin{aligned}
R & =\left[\begin{array}{ll}
0.1820 & 0.0099 \\
0.0099 & 0.0015
\end{array}\right], \\
\bar{H} & =\left[\begin{array}{ccc}
1.0658 & 51.8549 & -0.1291 \\
-0.1255 & -2.1491 & 0.0092
\end{array}\right]^{T} .
\end{aligned}
$$




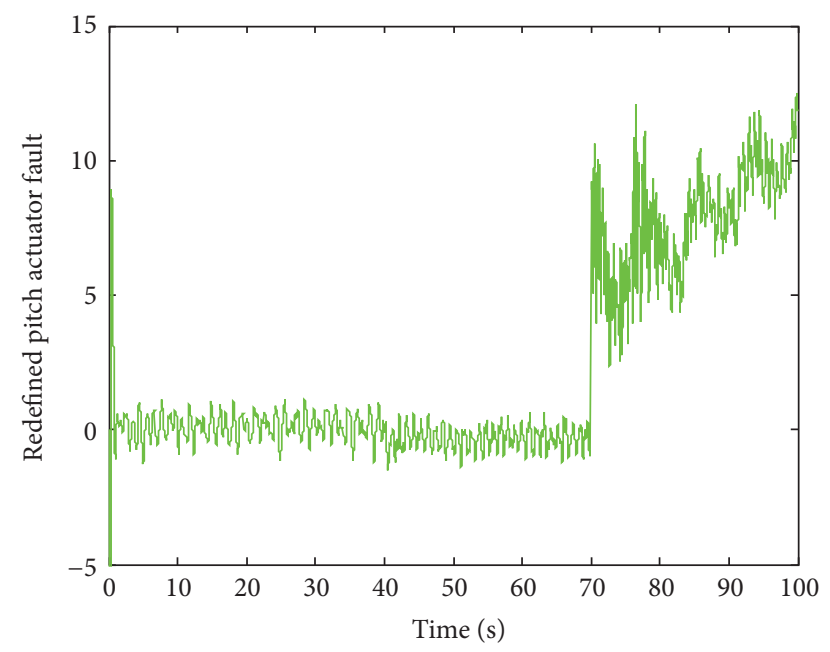

FIGURE 2: Estimate of newly defined pitch actuator fault $f_{a}$ with $f_{s}$ $=-0.2$.

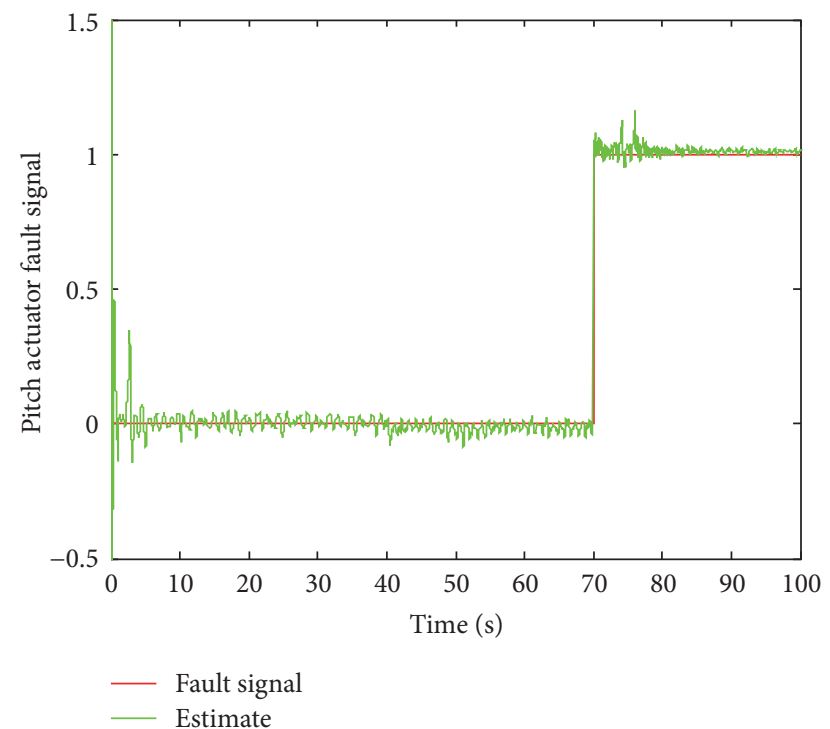

Figure 3: Pitch actuator original fault $\theta_{f}$ and its estimate with $f_{s}=$ -0.2 .

We select the matrices $G$ and $K$ as follows:

$$
\begin{aligned}
G & =\left[\begin{array}{ll}
0 & 0.0081
\end{array}\right], \\
K & =10^{-4} \times\left[\begin{array}{ll}
0.0174 & -0.3215
\end{array}\right] .
\end{aligned}
$$

The norm controller (53) has the following parameters: $b_{0}=-0.03, K_{P}=5.5$, and $K_{D}=5$.

The simulation results are shown in Figures 2-9. The estimation of the pitch actuator fault signal $f_{a}$ is redefined according to (9) and shown in Figure 2. However, one problem because of the redefined pitch actuator fault signal $f_{a}$ is that it is not easy to determine the severity of a fault because it is strongly coupled to the system states. The original pitch actuator fault signal can be constructed by using (10) and the reconstructed original fault is shown in Figure 3. Figures 3-5 show that the proposed descriptor sliding mode observer can

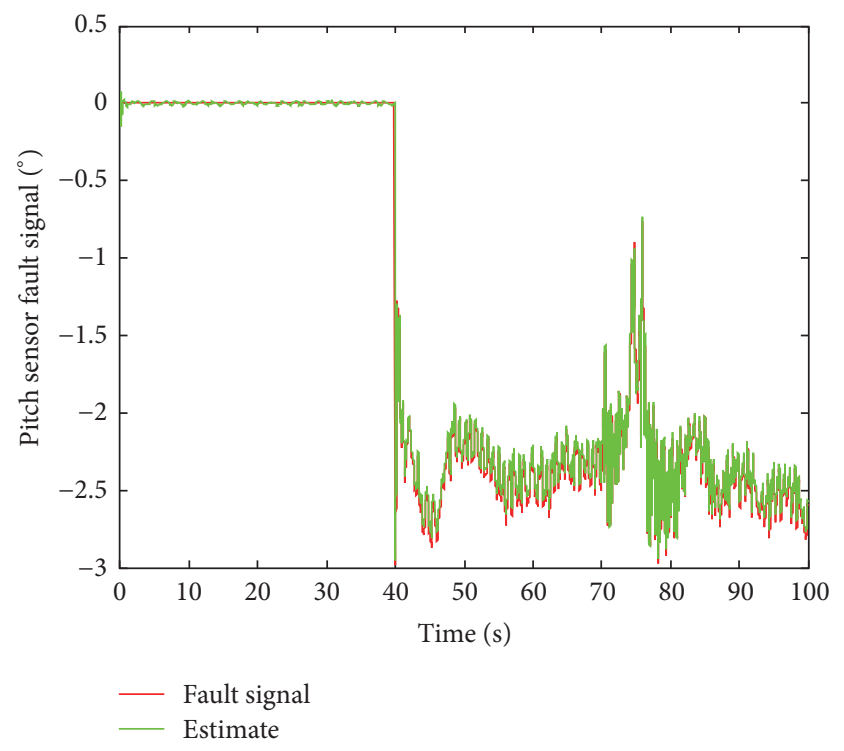

Figure 4: Pitch sensor fault and its estimate with $f_{s}=-0.2$.

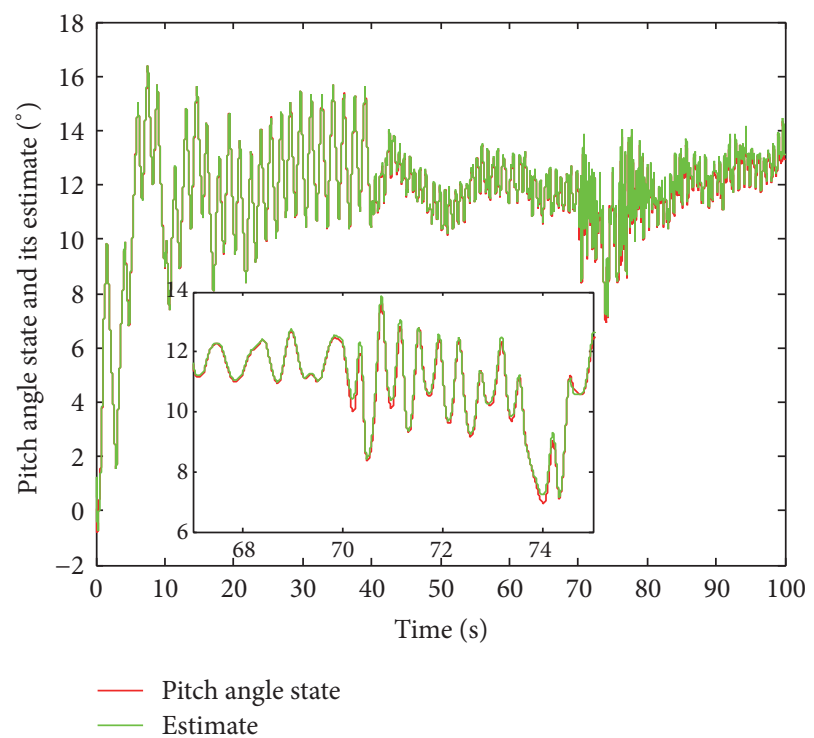

FIgURE 5: Pitch angle and its estimate with $f_{s}=-0.2$.

obtain a satisfactory estimation of the new augmented pitch system states. With the estimated pitch system augmented states, FTC is carried out with the strategy shown in (43). The FTC results are presented in Figures 6-9. In this part, as for the fault-tolerant scene of the fault-tolerant controller, the conventional controller is in a divergence state due to the worst case of the fault, so the corresponding effect contrast diagram is not given.

\section{Conclusion}

In this paper, a new architecture for active FTC controller is proposed for a wind turbine pitch system with simultaneous pitch actuator and sensor faults based on fault estimation and reconstruction to maintain nominal pitching performance. 


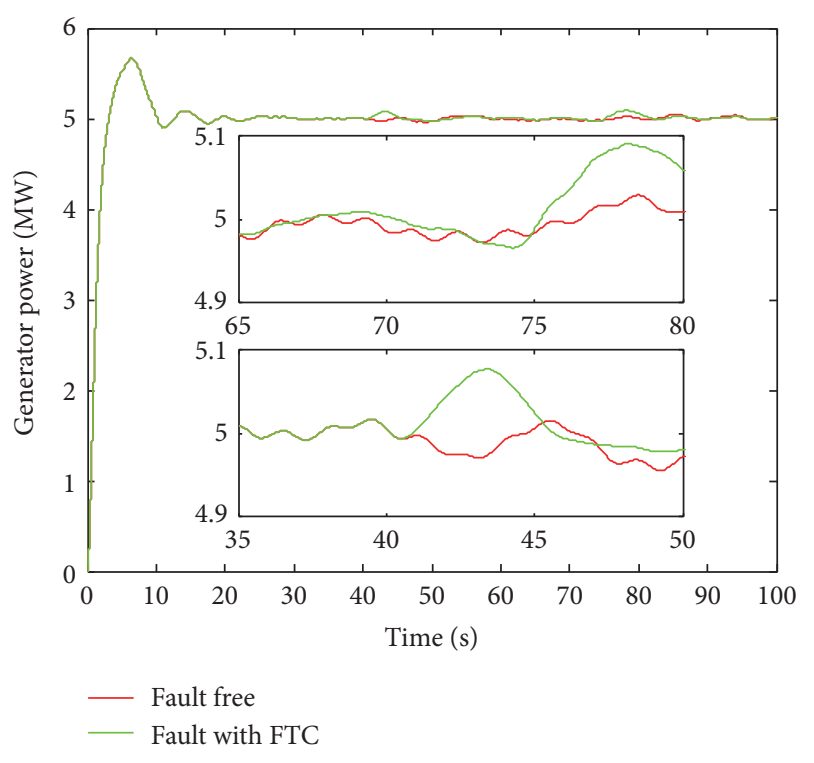

FIGURE 6: Generator power output with $f_{s}=-0.2$.
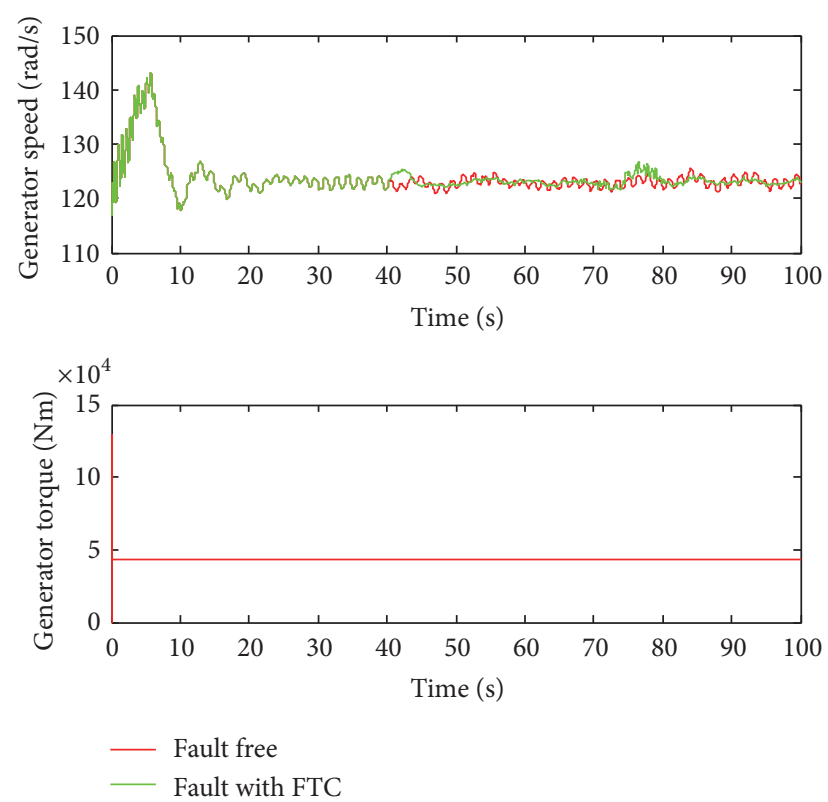

FIGURE 7: Generator speed and its torque with $f_{s}=-0.2$.

The FTC controller included both a normal controller, which was designed by using the active disturbance rejection control technique for estimating and compensating for the uncertainties, and a reconfigurable controller, which was designed by using the state and fault estimates obtained from an augmented descriptor sliding mode observer. The simulations performed in the FAST-MATLAB/Simulink demonstrate that the proposed FTC design can ensure the stability of the generator power output in the event of a fault or in cases without a fault.

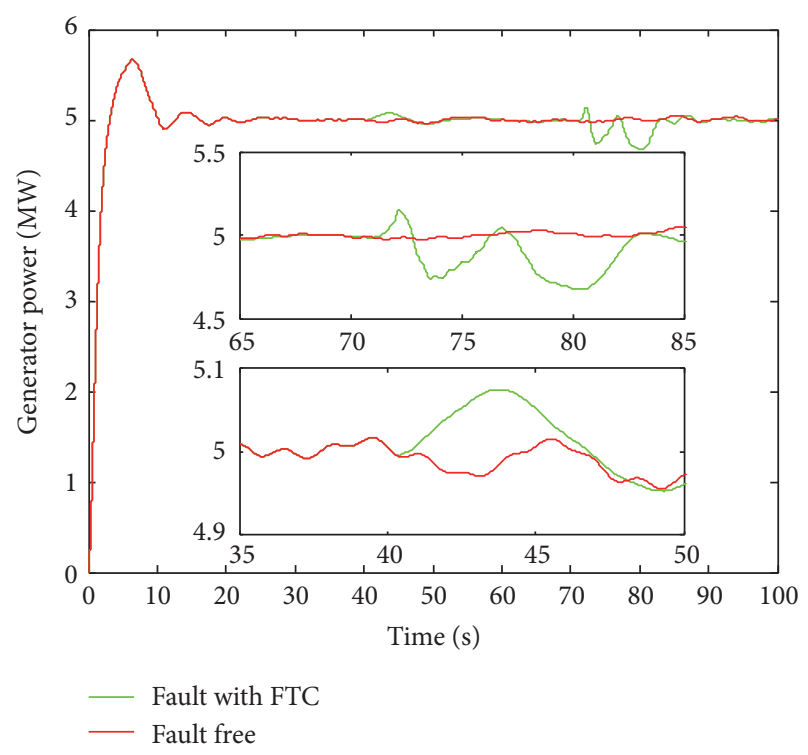

FIGURE 8: Generator power output with $f_{s}=-0.3$.
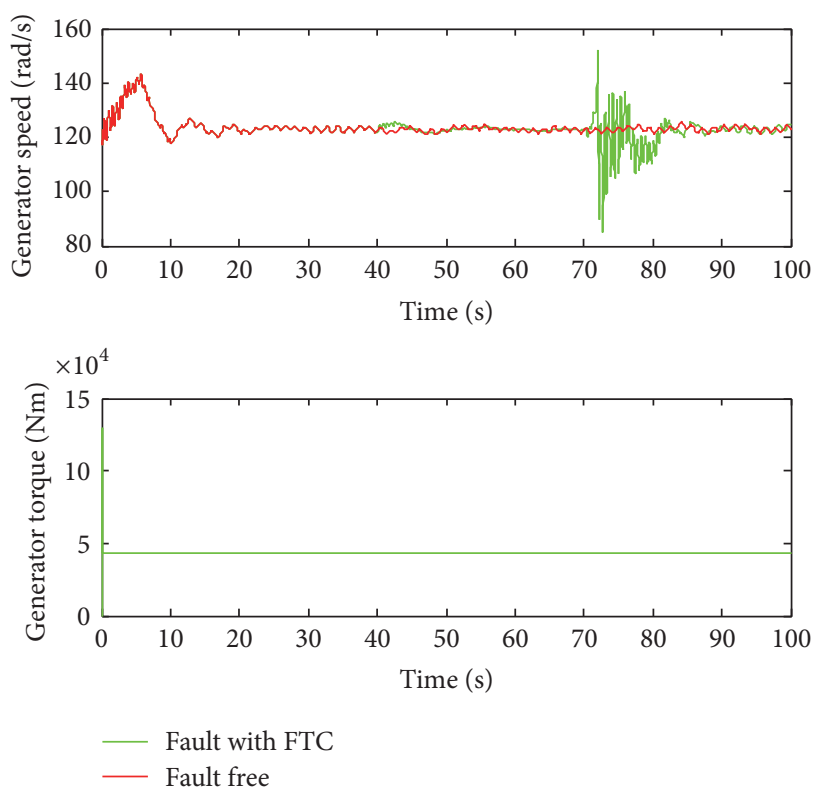

FIGURE 9: Generator speed and its torque with $f_{s}=-0.3$.

\section{Conflicts of Interest}

The authors declare that there are no conflicts of interest regarding the publication of this paper.

\section{Acknowledgments}

The National Natural Science Foundation of China (no. 51205046), Frontier and Applied Basic Research Projects (no. cstc2014jcyjA90005) funded by Chongqing Science and Technology Commission, The 56th Postdoctoral Science Foundation of China (no. 2014M562283), and Special Financial Grant (2015T80956) from the China Postdoctoral 
Science Foundation and Postdoctoral Special Projects (no. Xm2014003) funded by Chongqing Government are gratefully acknowledged.

\section{References}

[1] T. Burton, N. Jenkins, D. Sharpe, and E. Bossanyi, Wind Energy Handbook, Wiley, New York, NY, USA, 2001.

[2] T. Jain, J. J. Yame, and D. Sauter, "A novel approach to real-time fault accommodation in NREL's 5-MW wind turbine systems," IEEE Transactions on Sustainable Energy, vol. 4, no. 4, pp. 10821090, 2013.

[3] C. Sloth, T. Esbensen, and J. Stoustrup, "Robust and faulttolerant linear parameter-varying control of wind turbines," Mechatronics, vol. 21, no. 4, pp. 645-659, 2011.

[4] M. Sami and R. J. Patton, "Fault tolerant adaptive sliding mode controller for wind turbine power maximisation," in Proceedings of the 7th IFAC Symposium on Robust Control Design, ROCOND'12, pp. 499-504, Aalborg, Denmark, June 2012.

[5] H. Badihi, Y. Zhang, and H. Hong, "Wind turbine fault diagnosis and fault-tolerant torque load control against actuator faults," IEEE Transactions on Control Systems Technology, vol. 23, no. 4, pp. 1351-1372, 2015.

[6] P. F. Odgaard and J. Stoustrup, "Fault tolerant control of wind turbines using unknown input observers," in Proceedings of the 8th IFAC Symposium on Fault Detection, Supervision and Safety of Technical Processes, SAFEPROCESS 2012, pp. 313-318, Mexico City, Mexico, August 2012.

[7] J. Blesa, D. Rotondo, V. Puig, and F. Nejjari, "FDI and FTC of wind turbines using the interval observer approach and virtual actuators/sensors," Control Engineering Practice, vol. 24, no. 1, pp. 138-155, 2014.

[8] Y. Ren, L. Y. Li, J. Brindley, and L. Jiang, "Nonlinear PI control for variable pitch wind turbine," Control Engineering Practice, vol. 50, pp. 84-94, 2016.

[9] F. Shi and R. Patton, "An active fault tolerant control approach to an offshore wind turbine model," Journal of Renewable Energy, vol. 75, pp. 788-798, 2015.

[10] J. Lan, R. J. Patton, and X. Zhu, "Fault-tolerant wind turbine pitch control using adaptive sliding mode estimation," Journal of Renewable Energy, pp. 1-13, 2016.

[11] L. Chen, F. Shi, and R. Patton, "Active FTC for hydraulic pitch system for an off-shore wind turbine," in Proceedings of the 2nd International Conference on Control and Fault-Tolerant Systems, SysTol 2013, pp. 510-515, Nice, France, October 2013.

[12] M. Gálvez-Carrillo and M. Kinnaert, "Sensor fault detection and isolation in doubly-fed induction generators accounting for parameter variations," Journal of Renewable Energy, vol. 36, no. 5, pp. 1447-1457, 2011.

[13] M. S. Shaker and R. J. Patton, "Active sensor fault tolerant output feedback tracking control for wind turbine systems via T-S model," Engineering Applications of Artificial Intelligence, vol. 34, pp. 1-12, 2014.

[14] Y. Song, Y. Lu, and Z. Gan, "Descriptor sliding mode approach for fault/noise reconstruction and fault-tolerant control of nonlinear uncertain systems," Information Sciences, vol. 367368, pp. 194-208, 2016.

[15] K.-S. Lee and T.-G. Park, "An actuator fault reconstruction scheme for linear systems," Journal of Process Control, vol. 44, pp. 106-119, 2016.
[16] L. Chen, X. Huang, and S. Fu, "Observer-based sensor faulttolerant control for semi-Markovian jump systems," Nonlinear Analysis: Hybrid Systems, vol. 22, pp. 161-177, 2016.

[17] M. Liu and P. Shi, "Sensor fault estimation and tolerant control for Itô stochastic systems with a descriptor sliding mode approach," Automatica, vol. 49, no. 5, pp. 1242-1250, 2013.

[18] T. Wang, H. Gao, and J. Qiu, "A combined adaptive neural network and nonlinear model predictive control for multirate networked industrial process control," IEEE Transactions on Neural Networks and Learning Systems, vol. 27, no. 2, pp. 416425, 2016.

[19] O. Bagherieh and R. Nagamune, "Gain-scheduling control of a floating offshore wind turbine above rated wind speed," Control Theory and Technology, vol. 13, no. 2, pp. 160-172, 2015.

[20] J. Q. Han, "From PID to active disturbance rejection control," IEEE Transactions on Industrial Electronics, vol. 56, no. 3, pp. 900-906, 2009.

[21] J. Chen, L. Jiang, W. Yao, and Q. H. Wu, "Perturbation estimation based nonlinear adaptive control of a full-rated converter wind turbine for fault ride-through capability enhancement," IEEE Transactions on Power Systems, vol. 29, no. 6, pp. 27332743, 2014.

[22] L. Zhao, Y. Yang, Y. Xia, and Z. Liu, "Active disturbance rejection position control for a magnetic rodless pneumatic cylinder," IEEE Transactions on Industrial Electronics, vol. 62, no. 9, pp. 5838-5846, 2015.

[23] L. Chen, M. Liu, and S. Fu, "Adaptive sliding mode control for stochastic jump systems against sensor and actuator failures," IET Control Theory \& Applications, vol. 10, no. 16, pp. 20002009, 2016. 


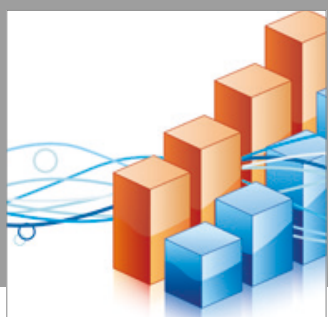

Advances in

Operations Research

vatersals

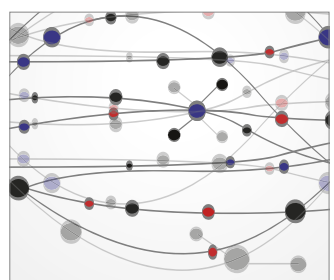

\section{The Scientific} World Journal
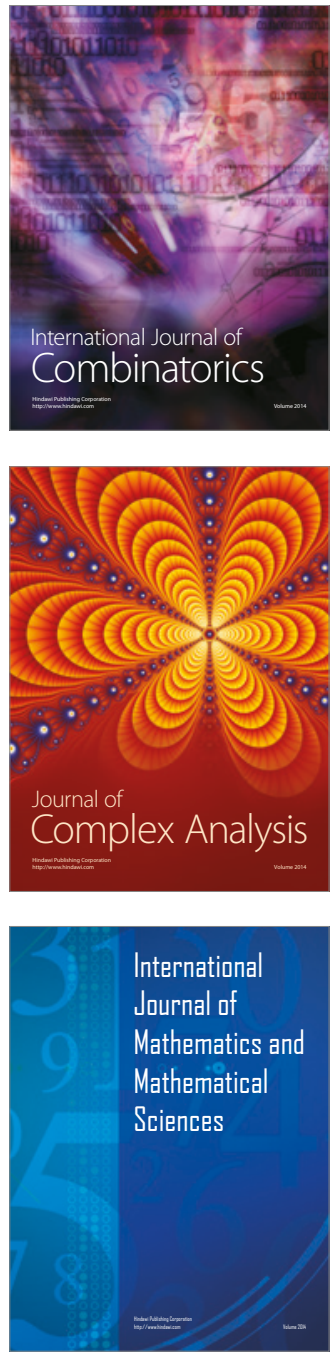
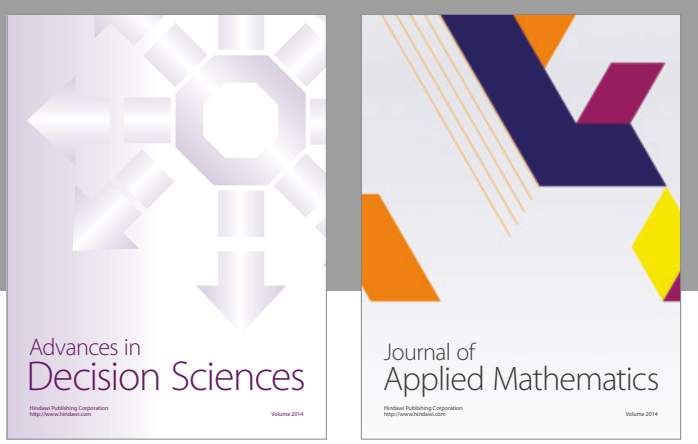

Algebra

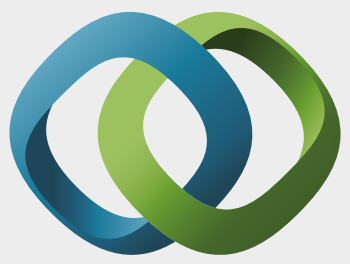

\section{Hindawi}

Submit your manuscripts at

https://www.hindawi.com
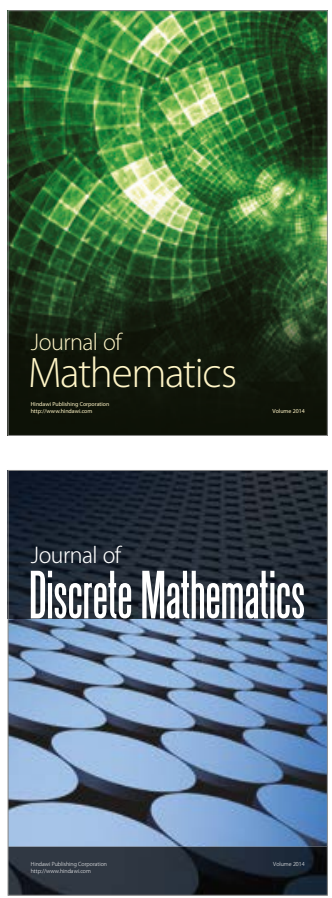

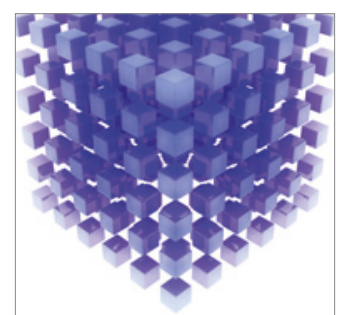

Mathematical Problems in Engineering
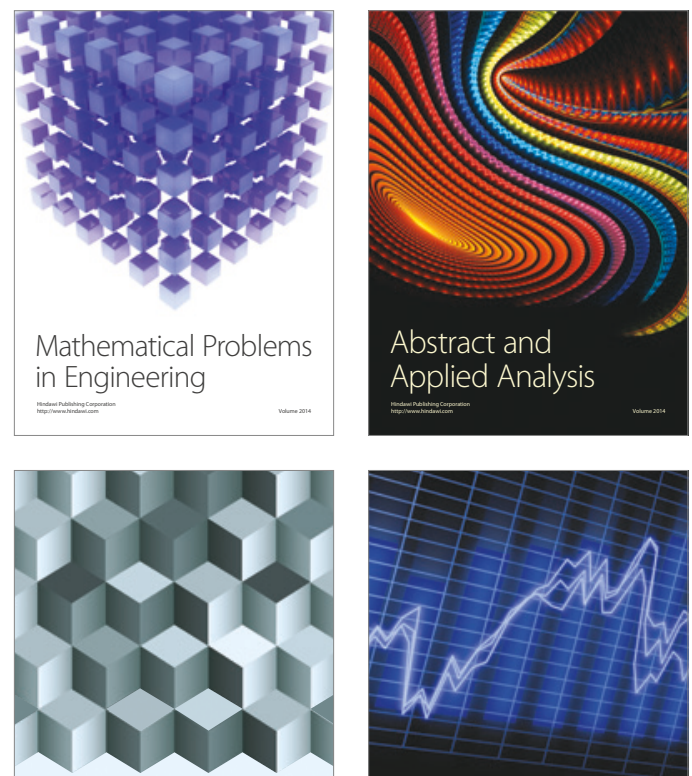

Journal of

Function Spaces

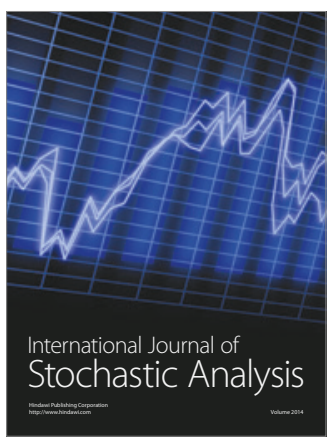

Probability and Statistics
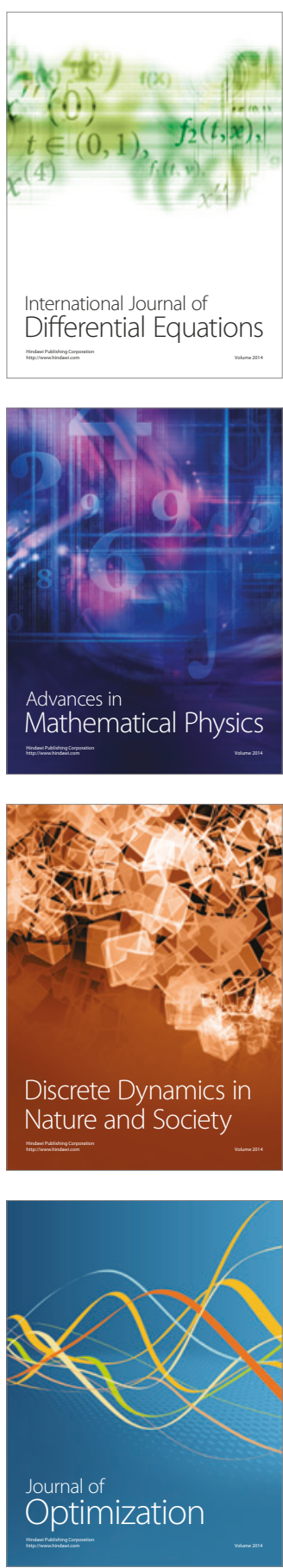